\title{
CFD BASED DETERMINATION OF SUBLIMATION MASS FLUX FOR LYOPHILIZATION INSIDE A VIAL
}

\author{
MATEJ ZADRAVEC ${ }^{1}$, ŽIGA ČASAR ${ }^{1}$, JURE RAVNIK ${ }^{1} \&$ MATJAŽ $^{1}$ HRIBERŠEK $^{1}$ \\ ${ }^{1}$ University of Maribor, Faculty of Mechanical Engineering, Slovenia.
}

\begin{abstract}
In the case of computational models of lyophilization in a vial, the intensity of drying is to a large extent controlled by the pressure conditions above the drying surface, as the drying driving force is the pressure difference between the saturation vapour pressure at the sublimation interface and the vapour partial pressure above the drying substance. In majority of studies, the effect of the vial and the rubber stopper geometry on the pressure conditions inside the vial is either neglected or taken into account by an estimated additional vapour pressure increase inside the vial. As the pressure conditions depend on the flow of sublimated solvent inside the vial-stopper conduit geometry, but are experimentally difficult to determine, a dedicated CFD analysis of flow conditions inside the vial-stopper channel was performed. The influence of imposing of the no-slip and slip conditions on the solid surfaces on the pressure drop in the system was studied and the effect of the increased partial pressure of the solvent on the sublimation rate was evaluated for the starting phase of the lyophilization by implementing the Stefan's one sided diffusion model. The computational results show, that the effect of the additional flow resistance due to the vial conduit and the stopper is most significant at lowest system temperatures, with as much as $30 \%$ increase in vapour pressure inside the vial.

Keywords: Computational fluid dynamics (CFD), heat and mass transfer, lyophilisation.
\end{abstract}

\section{INTRODUCTION}

In the process of lyophilization, the solvent is removed from the frozen mixture by the direct solid-gas phase change - sublimation of the solvent, which requires extremely low system pressure and system temperature in order to avoid transition of the frozen solvent into the liquid state. In case of lyophilization from a vial, the pressure conditions inside the vial determine the driving force for the sublimation, namely the pressure difference between the solvent saturation pressure at the sublimation front and the partial pressure of solvent vapor in the gas phase above the vial filling. The saturation pressure depends on the temperature, in this case the temperature in the material in the vial. The latter is directly influenced by the balance between the heat, supplied to the vial from the surroundings, mainly the shelf, and the heat, consumed by the sublimation process at the interface between the frozen material and the already de-iced porous part of the material. Additionally, the pressure conditions in the gas phase depend on the loading of the lyophilizer, the operating conditions in the condenser chamber, defining the lowest pressure level in the system, and on hydraulic resistance of the vial, the drying chamber and the condenser connecting channel with the valve.

In the case of lyophilization modeling, improving this pharmaceutical production process by means of numerical models gained significant interest in the last two decades, [1], [2], [3]. The majority of models, used in pharmaceutical engineering cases, are however developed for the case of a single vial. It is therefore extremely important to correctly set the boundary conditions for the heat and mass transfer numerical models. The boundary conditions present the link between the transport dynamics inside the vial filling, resolved by means of a dedicated lyophilization numerical model, and the exterior of the filling. The exterior typically includes the vial interior geometry, the vial stopper geometry, the interior geometry of the drying chamber, the condenser chamber and the connecting channel with the valve. The 
conditions outside the vial depend on heat and mass transfer conditions in the flow of sublimation vapor, which can computationally be resolved by a dedicated flow simulation by means of Computational Fluid Dynamics (CFD), which solves momentum conservation, energy conservation and species conservation equations for the fluid domain, resulting in spatial and temporal distribution of fluid velocity, pressure, temperature and partial vapor pressure. The latter information can therefore be directly used in the specification of the correct boundary conditions at the outlet from the vial.

As the lyophilization process is divided into two major parts, the primary drying and the secondary drying, the former presents the main challenge in setting the correct boundary conditions, as here the mass flux of the sublimated solvent is at largest in the whole process duration, especially at the start, when the frozen solvent is in direct contact with the gas in the vial [4].

This paper covers the modeling of the first part of the primary drying stage, with the aim to determine the interaction of the flow characteristics, with main focus on pressure distribution of evaporated solvent inside the vial, which is either open or partially stoppered, and the conditions of the sublimation mass flux from the vial filling.

\section{MODELING THE LYOPHILIZATION PROCESS}

The freeze-drying process is usually divided into two parts, the primary and secondary drying stage [5], [6], [7]. The process starts when the frozen substance is subjected to a sudden decrease in system pressure, setting the thermodynamic conditions below the triple point of the solvent (predominantly water) for sublimation to occur. The pressure difference between the sublimation surface and the vacuum induces sublimation, which due to the sublimation enthalpy acts as a strong energy sink. Heating of the shelves solves the problem of energy supply and enables to take control of the process. Because of the opened top of the vial, the drying starts at the top and proceeds toward the bottom of the vial. The first stage is therefore represented mainly by the sublimation process of the frozen liquid and it ends when all the frozen water is removed. After the first stage, the second stage begins, where the desorption dictates the drying process. The desorption process is also present in the primary drying stage in the dried region, but it is not a dominant process.

This paper covers the modeling of the primary drying stage, with the aim to determine the influence of the stopper on drying conditions at the surface of the filling at the beginning of the primary drying, when the frozen water is directly exposed to the interior of the vial.

\subsection{Computational model set-up}

Lyophilization is basically a freeze-drying process governed by the mass and energy conservation equations including the moving interface conditions. In the primary drying phase, when the frozen solvent is removed, the drying process depends on the pressure difference in solvent vapor pressures at the sublimation front and in the gas, filling the vial. Under isothermal conditions, the maximum drying rate occurs when the sublimation front is directly exposed to the surrounding gas, Fig. 1. As the drying proceeds, the sublimation front moves into the interior of the filling, with now the already ice-free porous region positioned between the sublimation front and the surrounding gas. In this part of the drying, the drying rate is reduced, as the porous region presents an additional mass transfer resistance, reducing the effective pressure difference for the drying process. In order to increase the drying rate, additional heat is supplied to the vial from the shelf, increasing the temperature and hence the vapour pressure at the sublimation front. When supplying heat, care must be taken not to exceed the melting 


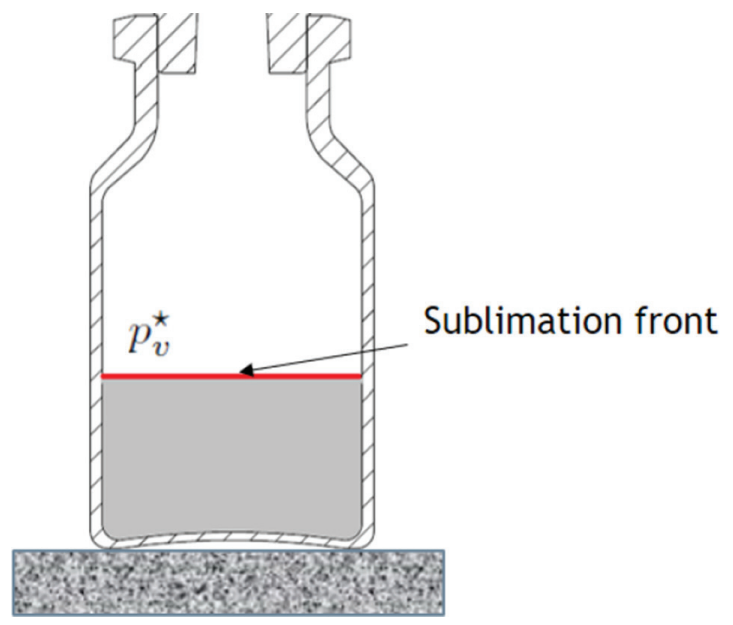

Figure 1: Sublimation position at the start or the primary drying phase.

point of the frozen solvent. In a computational set-up, this calls for a correct specification of boundary conditions for the solution of the coupled heat and mass transfer problem. The governing set of equations for the coupled problem consists of the energy conservation equation, written for the temperature field in the dried and the frozen region, the mass conservation equation written for the sublimating water in the form of bounded concentration in the porous part and the partial pressure of the solvent vapour, and for the inert gas in the form of partial pressure inside the dried region. The computational algorithm, described in [7], is an example of an effective model for the solution of the temperature and concentration fields of bounded solvent inside the substance in vial, together with solution of the partial pressure distribution of the inert gas and the solvent vapour inside the porous layer of the filling.

In order to evaluate the influence of the sublimation mass flux on the flow conditions inside the vial, either a dedicated experimental study or a computational study need to be performed. As the lyophilization takes place in a closed system of the lyophilizer under severe vacuum conditions, experimental study of velocity and pressure fields inside and outside of the vial is extremely difficult to perform. On the other hand, a computational study offers some major advantages, the most important being able to get a detailed temporal and spatial resolution of the velocity and especially pressure field inside the vial. In order to set-up a computational model, some simplifications have to be done. First, although in reality there are hundreds or even thousands of vials on a single shelf, the computational model will be focused on a single vial only, Fig. 2, in order to make a computational model reasonably fast, which would not be the case with thousands of vials. It is also expected that the main flow resistance comes from the flow of vapour inside the vial and through the stopper, as this geometry is the most confined one of the whole lyophilizer. Once the vapours exit the vial, the conduit cross sectional area increases significantly, leading to a much lower pressure drop as the one inside the vial. Secondly, the computational model is set for isothermal conditions, i.e. the temperature in the system is constant. Such conditions occur in the majority of the primary drying phase, when the supplied heat from the shelves balances the heat consumed by the sublimation process. For the case of non-isothermal conditions, the computational model would have to include also the computation of heat and mass transfer phenomena inside the vial filling. This could be done by means of a dedicated computational model, as 


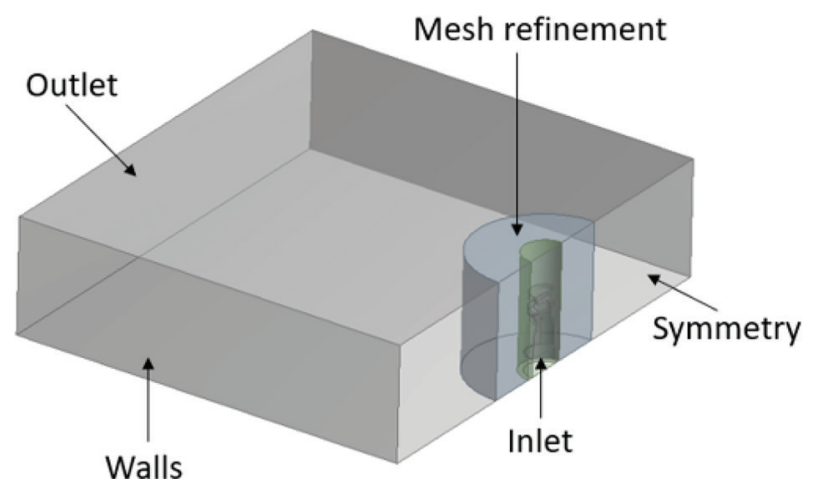

Figure 2: Computational fluid domain for the evaluation of hydraulic resistance of the vial.

for example models of [7] and [8], and a time marching solution procedure, which would significantly increase computational demands of the coupled model.

With majority of computational models of lyophilization in a vial, the set up of pressure boundary conditions at the top of the vial filling is connected directly to the saturation vapour pressure at the temperature of the condenser, operating at the lowest temperature level of the lyophilizer, [6], [7]. This would present an ideal case with no mass transfer resistance between the sublimation front and the condenser unit. On the other hand, since the stopper is seldom removed from the vial, its presence acts as an additional hydraulic resistance to the flow of the sublimated vapour, leading to increased pressure values for the evaporated solvent at the exit from the vial. As the stopper has a partial opening for the vapour flow when inserted in the vial, a CFD analysis of the vapour flow in the duct, formed by the interior of the vial together with the half inserted stopper as a typical stopper situation, offers an ideal way to determine pressure boundary conditions. These conditions would correspond to actual conditions inside the vial during lyophilization, and could therefore be used as a correct boundary conditions in the lyophilization model.

\subsection{Fluid flow model}

At the start of the primary drying, the gas inside the vial is almost entirely composed of the sublimated solvent [5]; therefore, the flow of the solvent vapour was solved by the CFD. As the computational tool, the Ansys CFD tools, the Ansys-CFX and the Ansys-Fluent were used, [12]. At the start, the Ansys-CFX model was used for the computational analysis, however due to the established differences in computational results between the no-slip an free slip boundary conditions a detailed analysis of the influence of the slip conditions needed to be done, leading to the use of the Ansys-Fluent code, with the option a detailed set-up of the velocity slip conditions at the walls.

The governing equations consisted of mass and momentum conservation for the incompressible fluid and were solved for the steady state case,

$$
\begin{gathered}
\vec{\nabla} \cdot \vec{u}=0, \\
(\vec{u} \cdot \vec{\nabla}) \vec{u}=-\frac{1}{\varrho} \vec{\nabla} p+v \Delta \vec{u}
\end{gathered}
$$


where $\vec{u}$ is the gas velocity, $v$ the gas kinematic viscosity, $\varrho$ the gas density and $p$ pressure.

Due to extremely low system pressure in a typical sublimation process, the rarefied gas effects can play an important role. In order to establish, whether the conditions inside the vial call for additional model modifications, the Knudsen number, defined as

$$
K n=\frac{\lambda}{L}
$$

with $\lambda$ the free molecular path and $L$ the system characteristic length, was evaluated. The molecular free path can be evaluated as [11]

$$
\lambda=\frac{k_{B} T}{\sqrt{2} \pi p d_{m}^{2}}
$$

with $k_{B}$ the Boltzmann constant, $d_{m}$ the diameter of the solvent molecule, in the case of water $d_{m}=2.7510^{-10} \mathrm{~m}, T$ the system temperature and $p$ the system pressure. For the system pressure of $13 \mathrm{~Pa}$ and the temperature of $243.15 K$, it follows

$$
\lambda=0.00086 m
$$

As the system characteristic length the inner diameter of the vial was selected, i.e. $L=20 \mathrm{~mm}$, yielding the value of $K n=0.044$. For the case of $T=253.15 K$, it follows $K n=0.0447$ and for $T=263.15 K$, the $K n=0.0465$. With this value of the Knudsen number, the molecular effects can play an important role [11] and the continuum mechanics models should be corrected. For the fluid flow in a duct under moderate Knudsen number values, the most common modification is to replace the no-slip condition at the walls with the slip condition. There exist several slip models [11], most notable the Navier model and the Maxwell model. The latter was selected in our case, as it does not require specification of some case dependent parameters. The original Maxwell model for the velocity slip along the wall direction reads as

$$
u_{s}=u_{g}-u_{w}=\left.\lambda \frac{d u}{d n}\right|_{n=0}
$$

with $u_{g}$ the gas velocity at the wall, $u_{w}$ the velocity of the wall and $\lambda$ the free molecular path. In the case of Ansys CFD model, the effect of velocity slip at the solid walls was evaluated within the CFD computations by the modified Maxwell model [12]:

$$
u_{w}-u_{g}=\left(\frac{2-a_{v}}{a_{v}}\right) K n \frac{\partial u}{\partial n}
$$

It has to be mentioned that the Maxwell model is only available in the Ansys-Fluent code and not in the Ansys-CFX. The model in Fluent is for the numerical use approximated as:

$$
u_{s}=\left(\frac{2-a_{v}}{a_{v}}\right) \frac{\lambda}{\delta}\left(u_{g}-u_{w}\right)
$$

with $\delta$ the distance from the wall to the center of the first cell. $\alpha_{v}$ is the momentum accommodation coefficient of the gas phase, and has a typical value of 0.45 for water vapour in a glass vial [13]. 


\subsection{Model of surface sublimation}

A dedicated model for the sublimation of the frozen solvent at the surface of the filling is needed in order to provide the inflow boundary condition for the CFD analysis of the flow inside the vial. Under the specified pressure conditions, one can assume that the main mass transfer mechanism for the solvent vapour inside the vial is diffusion, arising from pressure difference between the saturation pressure at the sublimation front, and the solvent vapour partial pressure in the vial surroundings. The saturation pressure depends on the temperature at the sublimation front as

$$
p_{v}^{*}=133.32 \cdot \exp \left(23.9936-\frac{2.19 \Delta H_{s}}{T}\right)
$$

with $\Delta H_{s}=2840.0 \mathrm{~kJ} / \mathrm{kg}$ for the calculated case of water as solvent.

The case of sublimation can be thus modeled as one-sided diffusion of water vapour from the layer of the frozen solvent in the direction of the vial opening, in the form of [9]:

$$
\vec{J}_{v}^{\prime}=\frac{C_{v}}{C_{v}+C_{i}} \vec{J}_{v}^{\prime}-D_{v, i} \vec{\nabla} C_{\nu}
$$

In the direction $z$, perpendicular to the ice surface layer the molar flux of vapour is

$$
J_{\nu}^{\prime}=\frac{C_{\nu}}{C_{\nu}+C_{i}} J_{\nu}^{\prime}-D_{v, i} \frac{d C_{\nu}}{d z} .
$$

By assuming that the temperature in the interior of the vial fluid volume is not varying significantly, and by using the ideal gas law

$$
P_{v}=C_{v} R T
$$

the molar flux (11) reads as

$$
J_{v}^{\prime}=\frac{p_{v}}{p_{v}+p_{i}} J_{v}^{\prime}-\frac{D_{v, i}}{R T} \frac{d p_{v}}{d z} .
$$

The vapour diffusivity in the binary mixture is taken according to [10] as:

$$
D_{v, i}=0.01883 \frac{\sqrt{T^{3}\left(\frac{1}{M_{v}}+\frac{1}{M_{i}}\right)}}{\left(p_{i}+p_{v}\right) \sigma_{v i}^{2} \Omega_{D}}
$$

The Lennard-Jones parameters for the binary mixture are [7]

$$
\sigma_{v i}=\frac{\sigma_{v}+\sigma_{i}}{2}, \quad \epsilon_{v i}=\sqrt{\epsilon_{v} \epsilon_{i}}
$$


with parameter $\Omega_{D}$ the collision integral. By inserting eqn. (14) into eqn. (13), it follows

$$
p_{i} J_{v}^{\prime}=-0.01883 \frac{\sqrt{T\left(\frac{1}{M_{v}}+\frac{1}{M_{i}}\right)}}{R \sigma_{v i}^{2} \Omega_{D}} \frac{d p_{v}}{d z} .
$$

To get the molar flux, integration is needed from the sublimation surface $\left(p_{v, 0}=p^{*}{ }_{v}\right)$ to the top of the vial with conditions of the free space of the lyophilizer drying chamber $\left(p_{v, h}\right)$,

$$
J_{v}^{\prime} \int_{0}^{h} d z=-0.01883 \frac{\sqrt{T\left(\frac{1}{M_{v}}+\frac{1}{M_{i}}\right)}}{p_{i} R \sigma_{v i}^{2} \Omega_{D}} \int_{p_{v, 0}}^{p_{v, h}} d p_{v}
$$

resulting in the final expression for the molar flux

$$
J_{v}^{\prime}=-0.01883 \frac{\sqrt{T\left(\frac{1}{M_{v}}+\frac{1}{M_{i}}\right)}}{p_{i} R \sigma_{v i}^{2} \Omega_{D}} \frac{p_{v, h}-p_{v, 0}}{h}
$$

The values of the applied parameters were: $M_{i}=29 \mathrm{kmol} / \mathrm{kg}, M_{v}=18 \mathrm{kmol} / \mathrm{kg}, T=230 \mathrm{~K}$, $p_{i}=4 P a, R=8314 \mathrm{~J} / \mathrm{kmol} K, \sigma_{\mathrm{v}, i}=3.754 \AA, \Omega_{D}=0.9332$. With known $J_{v}^{\prime}$ the sublimation mass flow rate can be computed as

$$
\dot{m}=M v J_{v}^{\prime}
$$

From eqn. (18) it is clear that the sublimation mass flow rate is a function of the outlet pressure from the vial $p_{v, h}$, which can be determined from the CFD analysis of the flow in the vial. As the case under consideration is the sublimation front positioned at the top of the filling, the calculated mass flow rate can be used to calculate the time, in which the top $2 \%(\Delta h)$ of the filling height (the layer of ice without porous matrix, see [7]) is removed by the process of sublimation, as

$$
\Delta t=\frac{\rho_{1} \Delta h}{M_{v} J_{v}^{\prime}}
$$

\subsubsection{Evaluation of the influence of the stopper}

The CFD analysis of the vial system was done in two consecutive steps. In the first step, the influence of the stopper on the flow conditions inside the vial was performed. For this case, the vial (denoted as A20-C13) with the outer diameter of $24 \mathrm{~mm}$, the inner diameter of $20 \mathrm{~mm}$, the height of $45 \mathrm{~mm}$ and the diameter of the opening of $12.6 \mathrm{~mm}$ was selected. The Ansys-CFX code was used for the simulation of the fluid flow in the system of the single vial on the shelf. As the Ansys-CFX code does not provide any of the advanced slip models, the two fundamental cases were considered: the case of with the no-slip conditions on the vial walls, and the extreme case of the slip - the free slip boundary condition, assuming a zero tangential stress at the solid boundary. Also, the flow inlet boundary condition at the sublimation front 


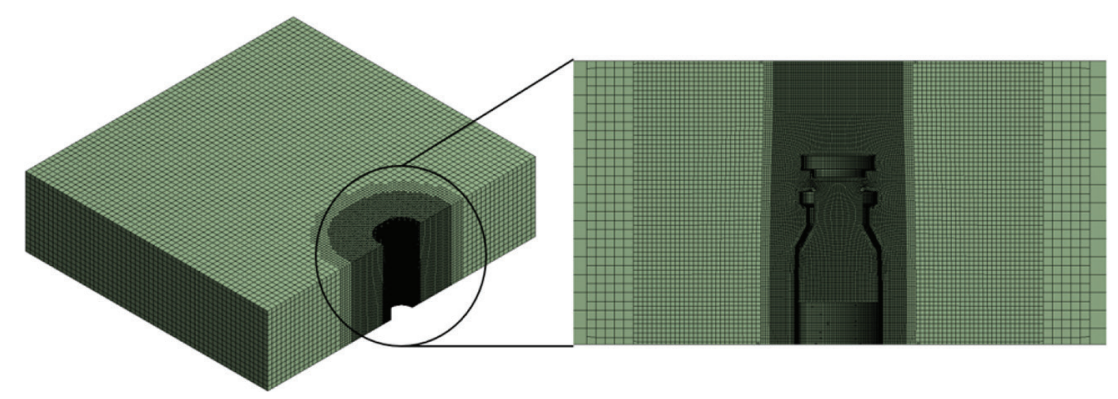

Figure 3: Computational grid for the surface sublimation-CFD flow coupled analysis (Ansys-Fluent case).

was set as constant and independent on the conditions in the interior of the vial. In order to specify physically relevant value of the mass flow, the maximum mass flow rate, obtained from experimental data in [8], was set at the sublimation surface. Also, the computational domain, shown in Fig. 2, was set in a way that the outlet from the domain was at one vial diameter distance from the centerline of the vial, in order to allow exiting of the vapour from the interior of the vial, but also to decrease the number of grid elements. The used mesh was a hybrid mesh, with tetrahedral elements inside the vial and hexahedral elements everywhere else. Also, a boundary layer was used at the vial walls. The mesh had 1, 287, 574 elements. The reference system pressure was set at $4 \mathrm{~Pa}$.

\subsubsection{Surface sublimation - CFD flow coupled analysis}

As already described, the Ansys-Fluent code was used to investigate the influence of the slip boundary conditions, since it has the Maxwell model already built-in. For the coupled simulation, a vial (denoted as A20-C8) was used, as this allowed using sublimation mass flow rate data from the previous study [7]. The vial had the outer diameter of $22 \mathrm{~mm}$, the height of $40 \mathrm{~mm}$ and the diameter of the opening of $12.6 \mathrm{~mm}$. The chosen vial had the same opening diameter as the vial A20-C13 and the same inner diameter, making it comparable to the A20C13 vial. The mesh was built using Ansys-Mesher, with the method CutCell, which resulted in 1,499,627 elements. A boundary layer at the vial walls has been considered, and a refinement was done near the vial, Fig. 3.

Since the mass flow of sublimation is dependent on the pressure level at the outlet from the vial, see eqn. (18), the imposing of the initial mass flow rate resulted in the CFD computation of the new level of vial outlet pressure, in turn reducing the mass flow rate of sublimation. An iterative computational procedure was therefore set-up for determining the mass flow rate, which could be achieved under the specified vial geometry and temperature condition (i.e. saturation pressure at the sublimation front). The iterative computational procedure is shown in Fig. 4.

\section{RESULTS AND DISCUSSION}

First, the influence of the stopper on the flow field inside the vial was studied by using the Ansys-CFX code. The system temperature was set at $230 K$, resulting in saturation pressure of $10.1 \mathrm{~Pa}$. The boundary condition at the outflow from the computational domain was set at $4 P a$. The computed pressure field inside the vial and its development through the stopper opening is shown in Fig. 5. The influence of the stopper is clearly visible, as the local velocity 


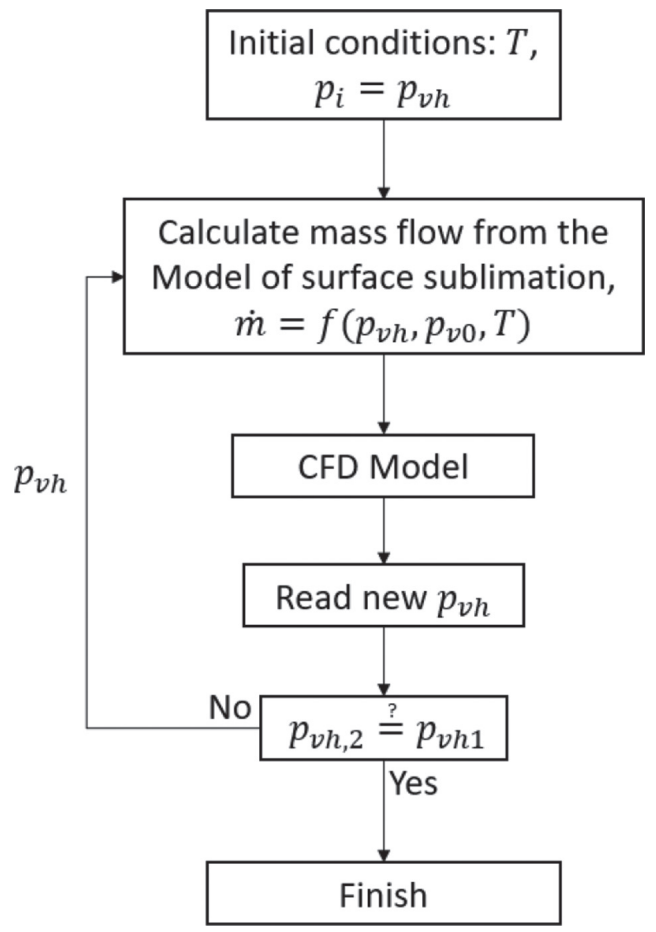

Figure 4: Iterative computational procedure for determination of the sublimation mass flow rate.

at the exit from the stopper increases significantly with regard to the condition in the vial throat. For the case of the free slip conditions, this effect is still evident, although the peak velocities are appr. 10\% lower. In order to evaluate the effect of the stopper, the case of the A20-C13 vial without inserted stopper was also computed. From the comparison of the
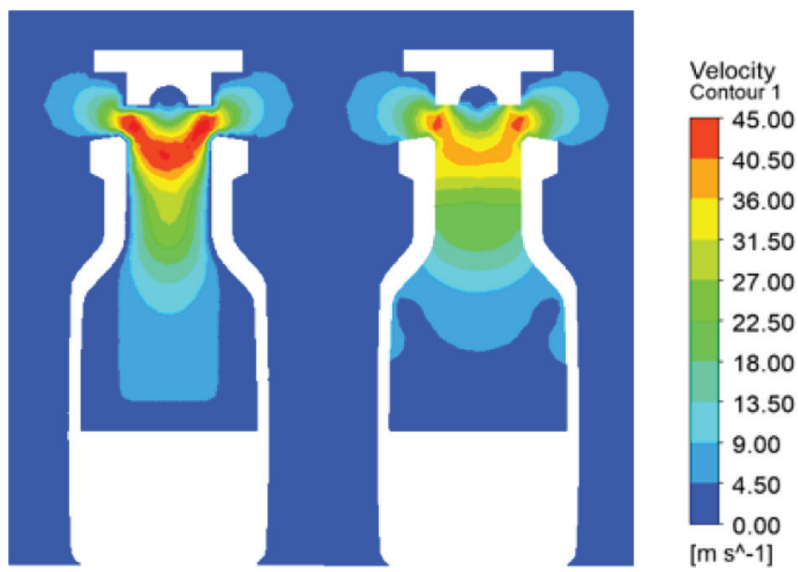

Figure 5: Velocity distribution inside the vial: no slip boundary condition (left), free slip boundary condition (right). 


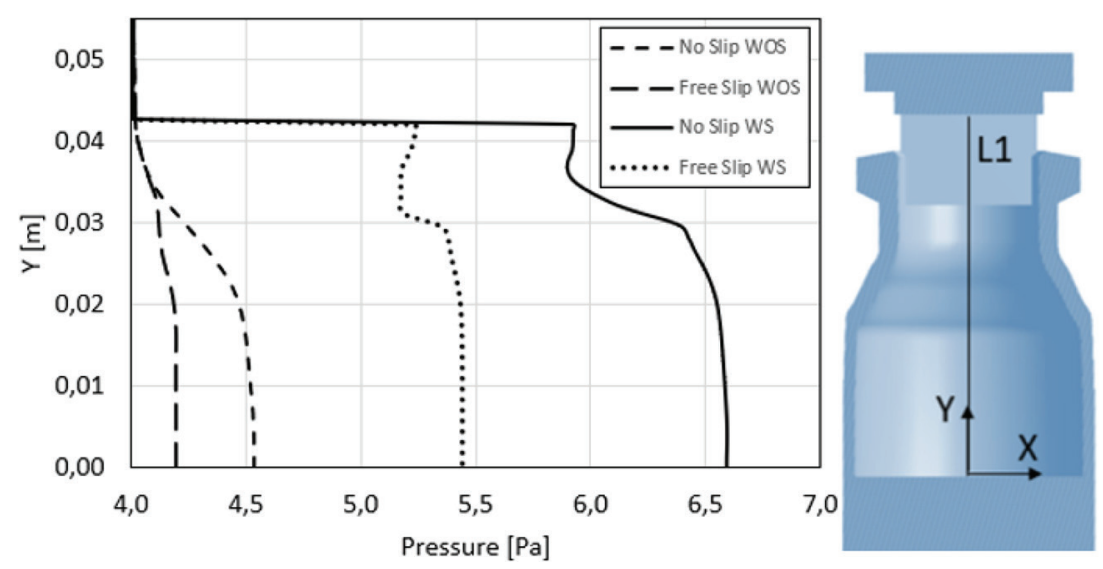

Figure 6: Pressure distribution inside the vial for the no-slip and free slip conditions at $-43^{\circ} \mathrm{C}$ and $4 \mathrm{~Pa}$ : WOS - without stopper, WS - with stopper.

pressure distribution along the centreline of the vial, Fig. 6, one can conclude that the presence of the stopper increases the pressure inside the vial by approx. $2 \mathrm{~Pa}$. It has to be noted though, that the applied computational grid was relatively coarse, resulting in non-smooth pressure development in the throat and stopper regions, which is especially evident in Fig. 6.

From the pressure distribution along the centerline of the vial one can now determine the pressure level at the exit from the vial (at $y=0.042 m$ for A20-C13) and consequently the pressure difference between the sublimation surface and the exit from the vial. In the case of the applied system pressure, the $1.8 \mathrm{~Pa}$ increase and the $1.3 \mathrm{~Pa}$ increase for the non-slip and free slip cases, respectively, present a significant rise in the pressure at the outlet from the vial, resulting in an increased mass transfer resistance. For the ideal case of only inert gas in the gas phase the pressure difference of $10.1 \mathrm{~Pa}$ would therefore lead to a decrease by up to $18 \%$ in the sublimation mass flow rate, eqn. (20). These preliminary results clearly indicate, that the stopper must therefore not be neglected in a vial lyophilization model.

In the performed preliminary analysis the sublimation temperature was very low, which corresponds to the beginning of the primary drying phase, when in the dryer with the frozen material in the vials the system pressure is drastically decreased. As in the rest of the primary phase the shelf temperature is increased, this increase transfers then also to the rise of the sublimation temperature, increasing the vapour pressure. With proceeding drying, the sublimation front moves inside the partially dried cake, and the sublimation vapour pressure increase is counterbalanced by the increased solvent vapour pressure in the porous region, which now presents the most important mass transfer resistance in the vial. To correctly model this process the fully coupled system of heat and mass transfer governing equation for the moving phase change problem, [7, 8], would have to be implemented in order to calculate the correct sublimation mass flow rate.

With conclusions from the preliminary test, the iterative computational procedure was implemented in the Ansys-Fluent for investigation of the effect of the wall boundary condition on flow development inside the vial. The computational grid used was refined in the interior of the vial and the stopper channels, in order to resolve high pressure and velocity gradients, especially at the vial walls, its accuracy important in the velocity slip boundary condition calculation, eqn. (8). The partial pressure of water vapour, pv, 0 , at the frozen 
solvent surface (inlet) was set as the saturation pressure, eqn. (9). Three different system conditions were computed: system pressure of $8 \mathrm{~Pa}$ at two temperature levels, $-20^{\circ} \mathrm{C}$ and $-30^{\circ} \mathrm{C}$, and system pressure of $13 \mathrm{~Pa}$ at temperature $-10^{\circ} \mathrm{C}$. The choice of lower system pressure with lower temperature levels is reasonable in order to assure larger solvent vapour pressure difference and hence drying rate. The calculated $p_{v, 0}$ for $10^{\circ} \mathrm{C}, 20^{\circ} \mathrm{C}$, and $30^{\circ} \mathrm{C}$ are 282.60Pa, 112.77Pa and 41.72Pa, respectively.

The computational results in form of the velocity profiles comparison for different wall boundary conditions and the system conditions of $-30^{\circ} \mathrm{C}$ and $8 \mathrm{~Pa}$ are shown in Fig. 7. The fluid velocity at the wall for the no slip boundary condition equals $0 \mathrm{~m} / \mathrm{s}$, for the Maxwell model $75.3 \mathrm{~m} / \mathrm{s}$, and for the free slip boundary condition $118 \mathrm{~m} / \mathrm{s}$. The Maxwell model computed velocity profile lies between the ones for the no slip and the free slip boundary condition, and the same is valid when comparing the pressure profiles along the centerline of the vial, Fig. 8. When comparing the latter profiles with profiles in Fig. 6, the effect of the much refined computational mesh inside the vial is clearly evident. Although the velocity profiles of the free slip and Maxwell slip model are significantly different, the pressure profiles are much closer together, Fig. 8.

The resulting mass flows are shown in Table 1 together with calculated drying times for the top $2 \%$ of the total vial filling height of $12 \mathrm{~mm}$, [7]. We can observe that the mass flow rate at a constant operating pressure of $8 \mathrm{~Pa}$ increases with increase in temperature, a result of the higher values of the saturation pressure at the sublimation front. Also, the mass flow rate increases with a higher degree of velocity slip at the solid wall, which is a consequence of a lower hydraulic resistance of the interior of the vial in the conditions of the flow at higher Knudsen number values. The lower hydraulic resistance leads to a decrease in the value of the solvent partial pressure $p_{v, h}$ at the outlet from the stopper, giving rise to higher solvent pressure difference and hence higher mass flow rates. In the case of the no slip condition, the pressure drop through the vial is the largest in all the computed cases, leading to the highest drying times. It is also interesting that the mass flow rates, computed for the two slip cases, are not significantly different, which leads to the conclusion that if the CFD code does not include a dedicated velocity slip model the choice of the free slip model in the case of moderate Knudsen number values is a better choice then implementation of the standard no-slip model.
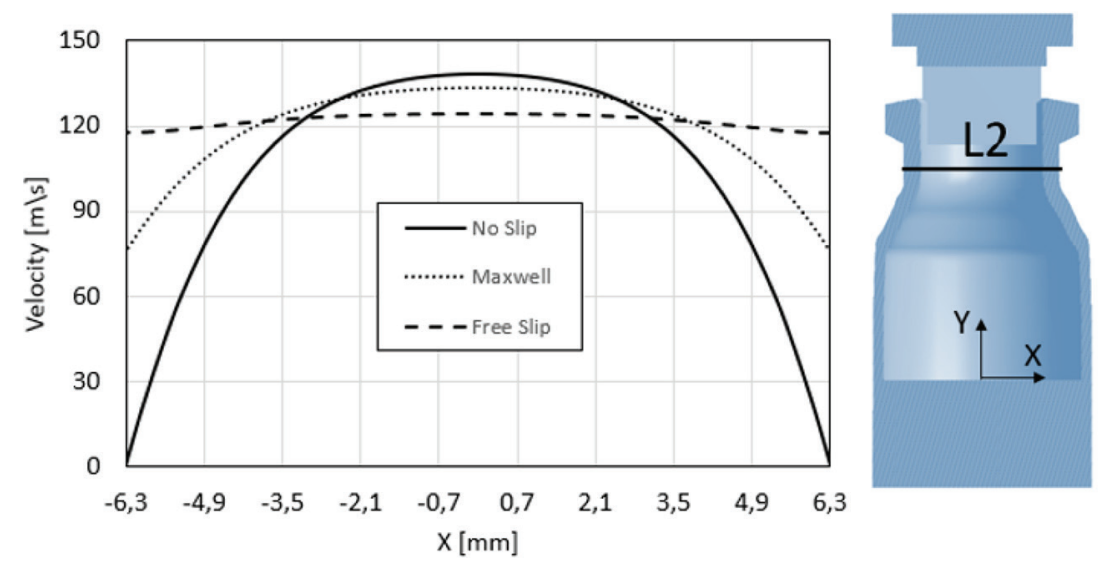

Figure 7: Velocity distribution inside the A20-C8 vial, for various wall boundary conditions at $-30^{\circ} \mathrm{C}$ and $8 \mathrm{~Pa}$. 


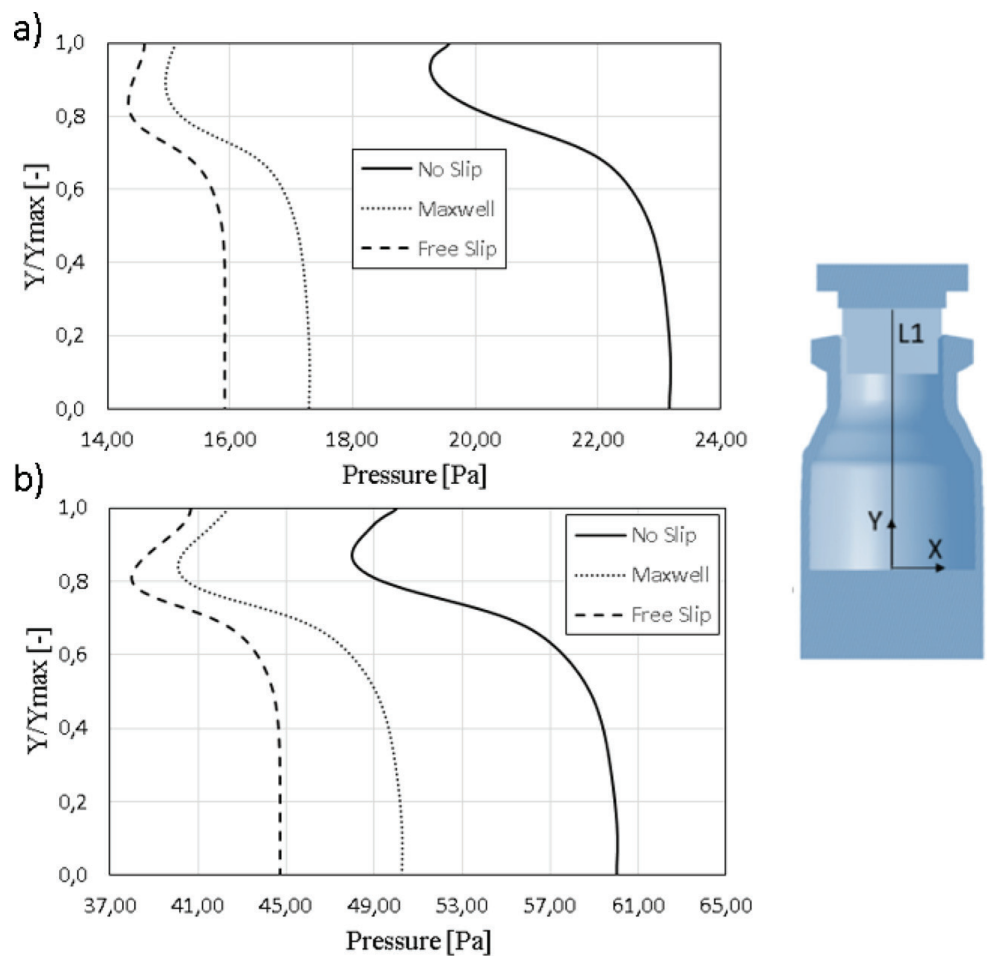

Figure 8: Pressure distribution inside the A20-C8 vial, for various wall boundary conditions at a) $-30^{\circ} \mathrm{C}$ and $8 \mathrm{~Pa}$ and b) $-20^{\circ} \mathrm{C}$ and $8 \mathrm{~Pa}$.

The presented computational cases highlight the fact that the influence of the vial and stopper geometry as well as the velocity slip conditions under conditions of higher values of Knudsen number should be considered when deriving an accurate computational model of lyophilization. It has to be mentioned that the presented cases are only simplified cases, as the temperature of the system was held constant. In the real case of lyophilization, especially at the start of the primary drying phase, high mass flow rates of sublimation lead to a large heat consumption at

Table 1: Resulting mass flows for various boundary and operating conditions.

\begin{tabular}{|c|c|c|c|c|c|}
\hline$T\left[{ }^{\circ} \mathrm{C}\right]$ & $p_{o p}[P a]$ & Bound. Condit. & $p_{v, h}[P a]$ & $\begin{array}{c}\text { Mass Flow } \\
{[\mathrm{kg} / \mathrm{s}]} \\
\end{array}$ & Time $[s]$ \\
\hline \multirow[t]{3}{*}{-30} & & No Slip & 19.80 & $2.040 \cdot 10^{-7}$ & 164.15 \\
\hline & 8 & Maxwell Free Slip & 15.04 & $2.490 \cdot 10^{-7}$ & 134.48 \\
\hline & & & 14.35 & $2.550 \cdot 10^{-7}$ & 131.32 \\
\hline \multirow[t]{3}{*}{-20} & & No Slip & 48.40 & $6.130 \cdot 10^{-7}$ & 54.63 \\
\hline & 8 & Maxwell Free Slip & 40.10 & $6.920 \cdot 10^{-7}$ & 48.39 \\
\hline & & & 38.07 & $7.110 \cdot 10^{-7}$ & 47.10 \\
\hline \multirow[t]{3}{*}{-10} & & No Slip & 76.00 & $1.234 \cdot 10^{-6}$ & 27.14 \\
\hline & 13 & Maxwell Free Slip & 58.70 & $1.337 \cdot 10^{-6}$ & 25.05 \\
\hline & & & 55.90 & $1.350 \cdot 10^{-6}$ & 24.80 \\
\hline
\end{tabular}


the sublimation front. Since in this phase of the drying the heat supply from the shelf is relatively low, this leads to cooling of the sublimation front, and to decrease of the saturation vapour pressure at the front, thus effectively reducing drying. In the case of the computational set up of the present study it is relatively easy to set up system conditions, for example the case for $-10^{\circ} \mathrm{C}$ and $8 P a$, that lead to computational results where the computed $p_{v, h}>p_{v, 0}$. This would not be possible when additional heat conservation equation of the drying material would be considered, which is the next step towards the complete computational analysis of the lyophilization process in a vial. It is also worth mentioning the computational cost of the analysis, which is typically in the order of a few CPU hours on a mid-range desktop computer. Although this presents no problem in the case of a single vial analysis, a direct use of a coupled CFD- vial lyophilization model for computing a typical lyophilization cycle (15-30 hours) would lead to a prohibitive large computational times, so a decoupled CFD analysis for the determination of vial boundary conditions only is the best way for the use of the proposed computational scheme.

The effect of increase of the pressure inside the vial can be additionally explained by analysing the experimental results, published in the reference [7]. In the article [7], one can see that in the early stages of lyophilization, the computed temperature inside the vial is lower than the experimental values, which is a consequence of a fixed water vapour pressure boundary conditions at the outlet from the vial, equal to the system pressure. This leads to an increase in sublimation flux, hence increasing the sublimation dependent heat sink at the sublimation interface, which directly results in a drop of the computed interface temperature. Hence, the increased value of the water vapour partial pressure set as the boundary condition will decrease the sublimation flux and consequently lead to increase of material temperature in this initial stage of the sublimation drying and better agreement with experimental observations.

\section{CONCLUSIONS}

The paper presents a CFD-based numerical model for determination of the pressure conditions inside the vial, which arise during the primary drying phase of lyophilization. The pressure increase inside the vial leads to a decrease in the pressure difference between the saturation pressure at the sublimation front and the solvent partial pressure inside the vial, which directly translates to a decrease in drying driving force. The consequence is an increase in drying time; therefore, the effect of the vial and stopper geometry and should not therefore be neglected when setting the correct outlet pressure boundary conditions for computation of lyophilization process inside the vial. The presented results for sublimation at different system temperatures in a vial under conditions of the first part of the primary drying, when the shelf temperature is increased from the freezing level, could be used also for estimating the pressure boundary conditions for the second part of the primary drying. In this case, the sublimation front moves into the interior of the vial filling, but the higher shelf temperature and the correspondingly higher saturation pressure at the sublimation front give rise to sublimation mass fluxes, comparable to the ones used in this study.

\section{REFERENCES}

[1] Brulls, M. \& Rasmuson, A. Heat transfer in vial lyophilization. International Journal of Pharmaceutics, 246, pp. 1-16, 2002. https://doi.org/10.1016/s0378-5173(02)00353-8

[2] Gan, K.H., Bruttini, R., Crosser, O.K. \& Liapis, A.I., Heating policies during the primary and secondary drying stages of the lyophilization process in vials: Effects of the arrangement of vials in clusters of square and hexagonal arrays on trays. Drying Technology, 22(7), pp. 1539-1575, 2004. https://doi.org/10.1081/drt-200025596 
[3] Daraoui, N., Dufour, P., Hammouri, H. \& Hottot, A., Model predictive control during the primary drying stage of lyophilisation. Control Engineering Practice, 18(5), pp. 483-494, 2010. https://doi.org/10.1016/j.conengprac.2010.01.005

[4] Hriberšek, M., Zadravec, M., Časar, Ž. \& Ravnik, J., The influence of the vial stopper on the flow and mass transfer conditions inside a vial. Wit Transactions on Engineering Sciences, 120, pp. 193-200, WIT Press, 2018. https://doi.org/10.2495/afm180191

[5] Pikal, M.J., Roy, M.L. \& Shah, S., Mass and Heat Transfer in Vial Freeze-Drying of Pharmaceuticals: Role of the Vial. Journal of Pharmaceutical Sciences, 73(9), pp. 1224-1237, 1984. https://doi.org/10.1002/jps.2600730910

[6] Mascarenhas, W.J., Akay, H.U. \& Pikal, M.J., A computational model for finite element analysis of the freeze-dyring process. Comput. Methods Appl. Mech. Engrg., 148, pp. 105-124, 1997. https://doi.org/10.1016/s0045-7825(96)00078-3

[7] Ravnik, J., Golobič, I., Sitar, A., Avanzo, M., Irman, Š., Kočevar, K., Cegnar, M., Zadravec, M., Ramšak, M. \& Hriberšek, M., Lyophilization model of mannitol water solution in a laboratory scale lyophilizer. Journal of Drug Delivery Science and Technology, 45, pp. 28-38, 2018. https://doi.org/10.1016/j.jddst.2018.02.015

[8] Ramšak, M., Ravnik, J., Zadravec, M., Hriberšek, M. \& Iljaž, J., Freeze-drying modeling of vial using BEM. Engineering Analysis with Boundary Elements, 77, pp. 145-156, 2017. https://doi.org/10.1016/j.enganabound.2017.01.011

[9] Bird, R.B., Stewart, W.E. \& Lightfoot, E.N., Transport Phenomena, 2007.

[10] Welty, J., Wicks, C., Wilson, R. \& Rorrer, G., Fundamentals Of Momentum, Heat, and Mass Transfer, John Wiley and sons, 2008.

[11] Kleinstreuer, C., Modern Fluid Dynamics, Springer Verlag, 2010.

[12] Ansys CFD, CFX 17.0, 2016.

[13] Scutella, B., Passot, S., Bourles, E., Fonseca, F. \& Trelea, I.C., How vial geometry variability influences heat transfer and product temperature during freeze-drying. Journal of Pharmaceutical Sciences, 106(3), pp. 770-778, 2017. https://doi.org/10.1016/j. xphs.2016.11.007 